\title{
Representation of Operators by Sampling in the Time-Frequency Domain
}

\author{
Monika Dörfler \\ Institut für Mathematik, Universität Wien, Alserbachstrasse 23 \\ A-1090 Wien, Austria \\ Monika.Doerfler@univie.ac.at \\ Bruno Torrésani \\ Université de Provence, LATP, UMR CNRS 6632, \\ CMI, 39 rue Joliot-Curie, 13453 Marseille cedex 13, France. \\ Bruno.Torresani@cmi.univ-mrs.fr
}

\begin{abstract}
Gabor multipliers are well-suited for the approximation of certain timevariant systems. However, this class of systems is rather restricted. To overcome this restriction, multiple Gabor multipliers allowing for more than one synthesis windows are introduced. The influence of the choice of the various parameters involved on approximation quality is studied for both classical and multiple Gabor multipliers. Fairly simple error estimates are provided, and the study is supplemented by numerical simulations. This paper is an extended and improved version of [6].
\end{abstract}

Key words and phrases: Operator approximation, time-frequency, Gabor multiplier, error estimates

2000 AMS Mathematics Subject Classification — 47B38, 47G30, 94A12, $65 \mathrm{~F} 20$.

\section{Introduction}

In signal processing, in particular speech and audio processing, the manipulation of given signals in the time-frequency domain is common practice, consider $[19,1,20]$ for some recent work. However, the operators that arise from these manipulations, so called time-frequency multipliers, have rather rarely been studied with a focus on the influence of the various involved parameters on the outcome of methods based on time-frequency analysis of signals.

In a recent paper [7], the authors describe the representation of operators in the time-frequency domain by means of a twisted convolution with the operator's spreading function. Although this description is not suitable for direct discretization, the spreading representation provides a better understanding of certain operators' behavior: it reflects the operator's action in the time-frequency 
domain. This motivates an approach that uses the spreading representation of time-frequency multipliers, in order to optimize the parameters involved. More specifically, in the one-dimensional, continuous-time case, given a linear operator $T$ on $\mathbf{L}^{2}(\mathbb{R})$ with integral kernel $\kappa_{T}$ and spreading function $\eta_{T}$ :

$$
\eta_{T}(b, \nu)=\int_{-\infty}^{\infty} \kappa_{T}(t, t-b) e^{-2 i \pi \nu t} d t
$$

we aim at modeling the operator by its action on the sampled short-time Fourier transform (STFT) or Gabor coefficients. The STFT of $f \in \mathbf{L}^{2}(\mathbb{R})$ is defined by

$$
\mathscr{V}_{g} f(b, \nu)=\langle f, \pi(b, \nu) g\rangle, \quad(b, \nu) \in \mathbb{R}^{2}
$$

where $\pi(b, \nu) g(x)=M_{\nu} T_{b} g=g(x-b) e^{2 \pi i \nu x}$ denotes the time-frequency shifted versions of a window $g \in \mathbf{L}^{2}(\mathbb{R})$. Sampling the STFT on a lattice $\Lambda$ then leads to the Gabor transform, which, for the sake of clarity, is denoted by $C_{g, \Lambda} f$ :

$$
C_{g, \Lambda} f(\lambda)=\langle f, \pi(\lambda) g\rangle, \quad \lambda \in \Lambda \subset \mathbb{R}^{2} .
$$

For the special case of a product lattice of the form $\Lambda=b_{0} \mathbb{Z} \times \nu_{0} \mathbb{Z}$, we obtain $C_{g, \Lambda} f(k, l)=\left\langle f, \pi\left(k b_{0}, l \nu_{0}\right) g\right\rangle=\left\langle f, M_{l \nu_{0}} T_{k b_{0}} g\right\rangle .{ }^{1}$ A good overview of Gabor analysis can be found in [12].

Purely multiplicative modification of the Gabor coefficients $C_{g, \Lambda} f(\lambda)$ leads to the definition of classical Gabor multipliers [11]. In this case, the linear operator applied to the coefficients is diagonal. Gabor multipliers provide accurate approximation of so-called underspread operators [18]. We will consider generalizations of the classical Gabor multipliers: the restriction to diagonality is relaxed in order to achieve better approximation for a wide class of operators at low cost. Moreover, in certain approximation tasks it is efficient, e.g. in the sense of sparsity, to use several side diagonals, but a lower redundancy (coarser sampling lattice $\Lambda$ ) in the Gabor system used. Further, the drawback resulting from coarse sub-sampling, i.e. large $b_{0}$ and/or $\nu_{0}$, can, to a certain extent, be compensated by using two or three instead of just one synthesis window. This case will be called multiple Gabor multiplier.

The aim of this contribution is the description of error estimates for the approximation of operators by multiple and generalized Gabor multipliers. The given error estimates are based on the operator's spreading function. The results give some insight in the choice of the parameters involved in approximation, in particular, of the windows and the lattice constants $b_{0}, \nu_{0}$.

This paper is organized as follows. Section 2 introduces Gabor multipliers and their generalizations. The basic idea of approximation in the spreading domain

\footnotetext{
${ }^{1}$ The finite-dimensional case $\mathcal{H}=\mathbb{C}^{L}$ is obtained similarly, replacing integrals with finite sums, and letting $k=0, \ldots N_{b}-1, l=0, \ldots N_{\nu}-1$, where $N_{b}=L / b_{0}, N_{\nu}=L / \nu_{0}$ and $b_{0}, \nu_{0}$ divide $L$.
} 
is explained. In Section 3, a general error estimate for the approximation of Hilbert-Schmidt operators by Gabor multipliers is derived, and several special cases are deduced thereof. As a noteworthy special case, the approximation of short-time Fourier multipliers by Gabor multipliers is considered. From these descriptions, guidelines for the choice of good parameters will be discussed in Section 4. Finally, Section 5 gives various insightful numerical experiments.

\section{Approximation in the time-frequency domain: the parameters}

Throughout this paper, $\mathcal{H}=\mathbf{L}^{2}(A)$ where $A$ is a locally compact abelian group. $\mathcal{H}$ is therefore equipped with an action of the Heisenberg group of time-frequency shifts, and corresponding versions of short time Fourier and Gabor transform may be defined. The standard cases $A=\mathbb{R}$ and $A=\mathbb{Z}_{L}, L \in \mathbb{N}$ will be of special interest to us.

\subsection{Gabor multipliers}

Let $g, h \in \mathcal{H}$, let $\Lambda$ be a lattice in the time-frequency space (see [9] for details on lattices and Gabor frames), and let $\mathscr{V}_{g}$ be the short time Fourier transform associated with analysis window $g$. To avoid confusions with the short time Fourier transform $\mathscr{V}_{g}$, we use the notation $C_{g, \Lambda}$ for the Gabor transform with window $g$ and lattice $\Lambda$ (the so-called analysis operator), and denote by $C_{g, \Lambda}^{*}$ the adjoint of $C_{g, \Lambda}$ (the synthesis operator). A Gabor multiplier [11] is defined as

$$
G_{m}: f \in \mathcal{H} \longmapsto G_{m} f=C_{h, \Lambda}^{*}\left(m \cdot C_{g, \Lambda} f\right) .
$$

Here, $m \cdot C_{g, \Lambda} f$ denotes the pointwise multiplication of $C_{g, \Lambda} f$ with the symbol $m \in \ell^{\infty}(\Lambda)$, Often $g=h$, i.e., analysis and synthesis window may be identical. For a given lattice $\Lambda$, we shall denote by $\Lambda^{\circ}$ the adjoint lattice [9], by $\Omega^{\circ}$ the corresponding fundamental domain, and by $\Pi^{\circ}$ the corresponding periodization operator. In the infinite-dimensional situation $\mathcal{H}=\mathbf{L}^{2}(\mathbb{R})$, and for a product lattice of the form $\Lambda=b_{0} \mathbb{Z} \times \nu_{0} \mathbb{Z}$, we have $\Lambda^{\circ}=t_{0} \mathbb{Z} \times \xi_{0} \mathbb{Z}$ with $t_{0}=1 / \nu_{0}$, $\xi_{0}=1 / b_{0}$, and $\Pi^{\circ} f(\zeta)=\sum_{\lambda^{\circ} \in \Lambda^{\circ}} f\left(\zeta+\lambda^{\circ}\right), \zeta \in \Omega^{\circ}$.

In a finite-dimensional setting $\mathcal{H}=\mathbb{C}^{L}$, with $\Lambda=\mathbb{Z}_{N_{b}} \times \mathbb{Z}_{N_{\nu}}$, with $N_{b}, N_{\nu}$ two divisors of $L$, we have $\Lambda^{\circ}=\mathbb{Z}_{L / N_{\nu}} \times \mathbb{Z}_{L / N_{b}}$, and the obvious form for the periodization operator.

In the definition of the multipliers, several parameters have to be fixed: the analysis and synthesis windows $g$ and $h$, the lattice $\Lambda$, and the symbol $m$. For practical as well as theoretical reasons, the windows should be well-localized in time and frequency. As for the lattice, it is expected that denser lattices will lead to better results in approximation, but higher computational cost. We will 
see, that the eccentricity of the lattice plays an important role in approximation quality and that lattices that exceed a certain density are not suitable.

Finally, the symbol $m$ can be optimized to best approximate a given operator. This problem was studied in [7] in the Hilbert-Schmidt setting. An explicit expression for the best Gabor multiplier approximation of an Hilbert-Schmidt operator $T$ (in the sense that the Hilbert-Schmidt norm $\left\|T-G_{m}\right\|_{H S}$ is minimized) was obtained in the spreading domain, see Theorem 1 . The spreading function of a Gabor multiplier $G_{m}$ takes the form $\eta_{G_{m}}(\zeta)=\widetilde{m}(\zeta) \cdot \mathscr{V}_{g} h(\zeta)$, where $\widetilde{m}$ is the symplectic Fourier transform of the sequence $m$ :

$$
\widetilde{m}(\zeta)=\mathcal{F}^{s} m(\zeta)=\sum_{\lambda \in \Lambda} m(\lambda) e^{2 i \pi[\lambda, \zeta]}, \quad \zeta \in A \times \hat{A} .
$$

Here, $[\cdot, \cdot]$ denotes the usual symplectic form (e.g., for the rectangular lattice $\Lambda=b_{0} \mathbb{Z} \times \nu_{0} \mathbb{Z}$, we have $\left.\left[\left(k b_{0}, l \nu_{0}\right),(t, \xi)\right]=l \nu_{0} t-k b_{0} \xi\right)$.

Note, that $\widetilde{m}$ is a $\Lambda^{\circ}$-periodic function and this periodicity has a decisive influence on the quality of operator representation by Gabor multipliers. Loosely speaking, since $\eta_{G_{m}}(\zeta)=\widetilde{m}(\zeta) \cdot \mathscr{V}_{g} h(\zeta)$, a given spreading function can only be accurately reproduced in one fundamental domain of $\Lambda^{\circ}$. Even for underspread operators whose spreading function's support is contained in $\Omega^{\circ}$, the periodicity of $\widetilde{m}$ leads to aliasing effects, since $\mathscr{V}_{g} h$ is never compactly supported (see [14] for a discussion of STFT-related uncertainty inequalities). This phenomenon is illustrated in Figure 1, where an example of an operator with symmetric spreading function is shown. It is obvious that the amount of aliasing depends on the window decay, so, as mentioned before, localized windows must be chosen.

Given the spreading function of the operator to be approximated, the above observations give immediate insight in how the parameters involved in the approximation of operators by Gabor multipliers have to be chosen. Generally speaking, good approximation by a classical Gabor multiplier is possible, if the essential support of the spreading function is contained in the fundamental domain $\Omega^{\circ}$ of the adjoint lattice for a dense enough lattice $\Lambda$. In this case, to reduce aliasing as much as possible, the analysis and synthesis windows must be chosen such that $\mathscr{V}_{g} h$ is small outside $\Omega^{\circ}$ and positive on the support of the spreading function, also see Section 4.1.

\subsection{Generalizing Gabor multipliers}

In order to extend the good approximation quality of Gabor multipliers to a more general class of operators, we may allow for more sophisticated action on the time-frequency coefficients. In particular, instead of using just diagonal matrices, we may introduce several side-diagonals. This idea leads to the following definition, by considering the special case $h^{(j)}=\pi\left(\mu_{j}\right) h$ in the definition below. Generally speaking, multiple Gabor multipliers are sums of Gabor multipliers with different synthesis windows and symbols. 

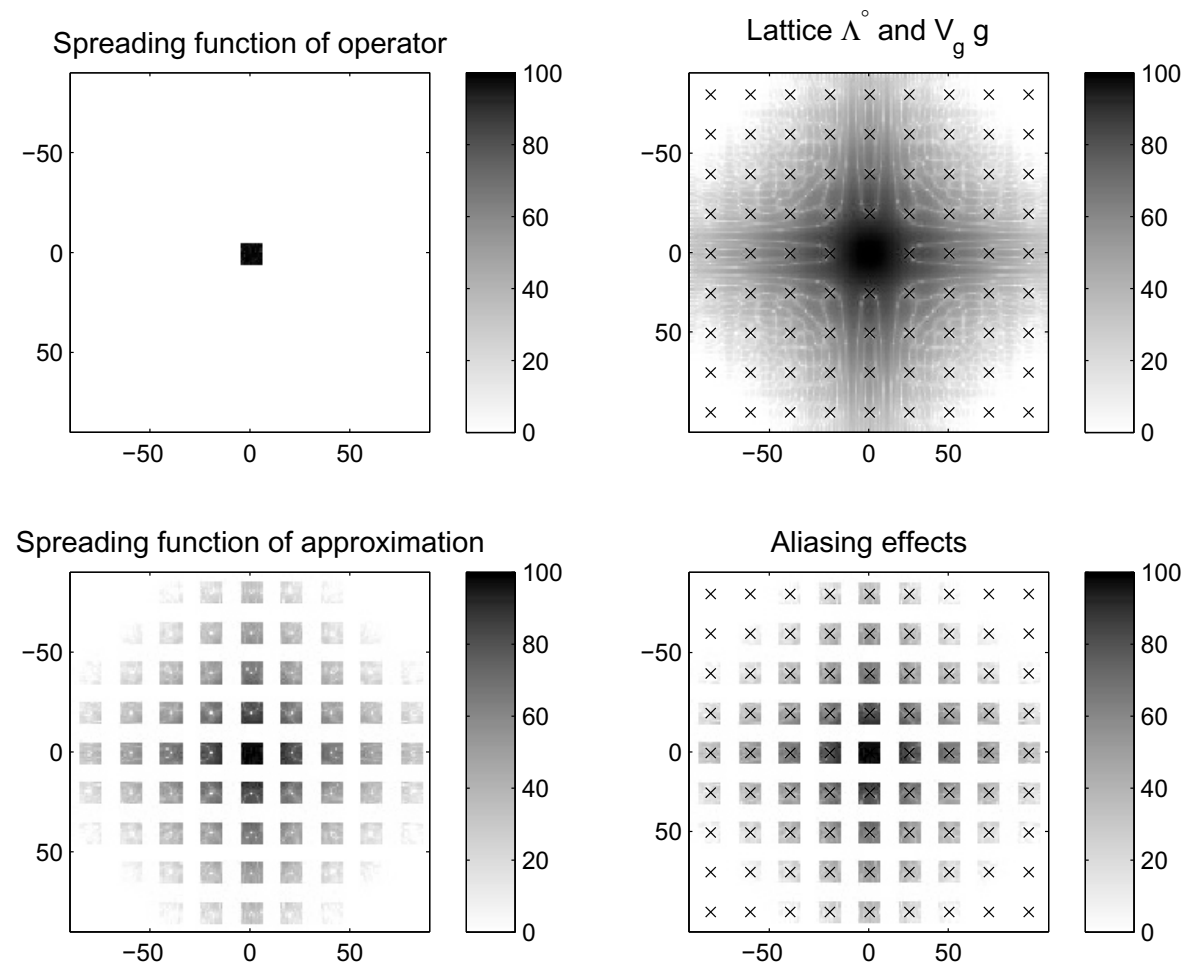

Figure 1: Example showing the aliasing effect caused by approximation with Gabor multiplier for an operator with compactly supported spreading function (top left). The adjoint lattice $\Lambda^{\circ}$ and the windows' $S T F T \mathscr{V}_{g}$ h are shown superimposed (top right). The approximation's spreading function alone and with $\Lambda^{\circ}$ are shown in the lower plots.

Definition 1 (Multiple and generalized Gabor Multiplier). Let $g, h^{(j)} \in \mathcal{H}$, for $j=1, \ldots, J$ denote an analysis and a set of synthesis window functions. Let $\Lambda$ be a time-frequency lattice.

1. For $\mathbf{m}=\left\{m_{j} \in \ell^{\infty}(\Lambda), j \in J\right\}$, a family of bounded functions on $\Lambda$, the associated multiple Gabor multiplier $G_{\mathbf{m}}$ is defined as follows: for all $f \in \mathcal{H}$

$$
G_{\mathbf{m}} f=\sum_{\lambda \in \Lambda} \sum_{j \in J} m_{j}(\lambda)\langle f, \pi(\lambda) g\rangle \pi(\lambda) h^{(j)} .
$$

2. A generalized Gabor multiplier is a multiple Gabor multiplier whose synthesis window functions are time-frequency shifted copies $h^{(j)}=\pi\left(\mu_{j}\right) h$, for a finite set of time-frequency shifts $\left\{\mu_{j}, j \in J\right\}$ of a unique window function $h$.

Note that the spreading function of multiple Gabor multipliers is given by $\eta_{G_{\mathbf{m}}}=\sum_{j} \widetilde{m}_{j} \mathscr{V}_{g} h^{(j)}$. 
The operator approximation with multiple Gabor multipliers involves, in addition to the choice of the parameters mentioned above, the choice of the analysis windows or the sampling points $\mu_{j}$. Approximation by sums of Gabor multipliers in the operator norm was treated in [15], with an application to the modeling of channel matrices in OFDM. Since the latter are assumed to be invertible, Hilbert-Schmidt norm approximation does not apply.

\section{Error analysis in $\mathbf{L}^{2}(\mathbb{R})$}

Multiple and generalized Gabor multipliers were introduced in [7]. For the sake of completeness, let us restate the corresponding approximation result, with the notations of the present paper. Given analysis and synthesis windows $g \in \mathbf{L}^{2}(\mathbb{R})$ and $h^{(j)} \in \mathbf{L}^{2}(\mathbb{R}), j=1, \ldots J$, introduce the $\Lambda^{\circ}$ periodizations:

$$
U_{j j^{\prime}}=\Pi^{\circ}\left(\mathscr{V}_{g} h^{\left(j^{\prime}\right)} \overline{\mathscr{V}_{g} h^{(j)}}\right), \quad B_{j}=\Pi^{\circ}\left(\eta_{H} \overline{\mathscr{V}_{g} h^{(j)}}\right), \quad j, j^{\prime}=1, \ldots J
$$

Denote by $\mathbf{U}$ the matrix-valued function with matrix elements $U_{j j^{\prime}}$ and by $\mathbf{B}$ the vector with components $B_{j}$.

Theorem 1. Let $g \in \mathcal{H}$ and $h^{(j)} \in \mathcal{H}, j=1, \ldots J$ be such that for almost all $\zeta \in \Omega^{\circ}$, the matrix $\mathbf{U}(\zeta)$ is invertible.

Let $T$ be an Hilbert-Schmidt operator on $\mathcal{H}$, with spreading function $\eta_{T}$. Then the vector $\mathbf{m}=\left(m_{1}, \ldots m_{J}\right)$ of symbols of the multiple Gabor multiplier that minimizes the approximation error $\left\|T-G_{\mathbf{m}}\right\|_{H S}$ is obtained from the solution of the matrix equation

$$
\widetilde{\mathbf{m}}(\zeta)=\mathbf{U}(\zeta)^{-1} \cdot \mathbf{B}(\zeta), \quad \zeta \in \Omega^{\circ}
$$

where $\widetilde{\mathbf{m}}=\left(\widetilde{m}_{1}, \ldots \widetilde{m}_{J}\right)$ is the vector of symplectic Fourier transforms of $\mathbf{m}$.

The invertibility condition for $\mathbf{U}(\zeta)$ (which reduces to the classical one $[2,7]$ in the Gabor multiplier case), is equivalent to linear independence of the system of projection operators involved, see [7] for details. Obviously, for a very dense lattice $\Lambda$ (i.e., high redundancy), $\Lambda^{\circ}$ is very coarse and this property is usually not fulfilled.

The case of a unique synthesis window may be immediately obtained from the above formula. Note that formula (5) allows for an efficient implementation of the otherwise expensive calculation of the best approximation by multiple Gabor multipliers, compare [8] for an algorithm that applies to the classical Gabor multiplier situation.

We may now give an expression for the error in the approximation given above, in the case $\mathcal{H}=\mathbf{L}^{2}(\mathbb{R})$. We define, for the Hilbert-Schmidt operator $T$,

$$
\Gamma_{T}=\Pi^{\circ}\left(\left|\eta_{T}\right|^{2}\right) .
$$


Proposition 1. Let $T$ be a Hilbert-Schmidt operator on $\mathcal{H}=\mathbf{L}^{2}(\mathbb{R})$, let $\widetilde{\mathbf{m}}$ denote the vector-valued function obtained as in (5) and let $\mathbf{m}$ be its inverse symplectic Fourier transform. Then the approximation error $E=\left\|T-G_{\mathbf{m}}\right\|_{H S}^{2}$ is given by

$$
E=\int_{\Omega^{\circ}} \Gamma_{T}(\zeta)\left(1-\frac{\sum_{i, j}\left(\mathbf{U}^{-1}\right)_{i j}(\zeta) B_{i}(\zeta) \overline{B_{j}}(\zeta)}{\Gamma_{T}(\zeta)}\right) d \zeta
$$

Proof. For simplicity, we set $\mathscr{V}_{j}=\mathscr{V}_{g} h^{(j)}$. Since the mapping $T \mapsto \eta_{T}$ is unitary, see [10], we may start from

$$
\begin{aligned}
\left\|H-G_{\mathbf{m}}\right\|_{H S}^{2} & =\left\|\eta_{T}-\sum_{j} \widetilde{m}_{j} \mathscr{V}_{g} h^{(j)}\right\|^{2} \\
& =\left\|\eta_{T}\right\|^{2}-2 \operatorname{Re}\left(\sum_{j}\left\langle\eta_{T}, \widetilde{m}_{j} \mathscr{V}_{j}\right\rangle\right)+\operatorname{Re}\left(\sum_{j, j^{\prime}}\left\langle\widetilde{m}_{j} \mathscr{V}_{j}, \widetilde{m}_{j^{\prime}} \mathscr{V}_{j^{\prime}}\right\rangle\right)
\end{aligned}
$$

and find by a straight-forward calculation that

$$
\sum_{j}\left\langle\eta_{T}, \widetilde{m}_{j} \mathscr{V}_{j}\right\rangle=\sum_{j, j^{\prime}} \int_{\Omega^{\circ}}\left(\mathbf{U}^{-1}\right)_{j j^{\prime}}(\zeta) B_{j}(\zeta) \overline{B_{j^{\prime}}}(\zeta) d \zeta=\sum_{j} \int_{\Omega^{\circ}} \widetilde{m}_{j}(\zeta) \overline{B_{j}}(\zeta) d \zeta
$$

whereas

$$
\sum_{j, j^{\prime}}\left\langle\widetilde{m}_{j} \mathscr{V}_{j}, \widetilde{m}_{j^{\prime}} \mathscr{V}_{j^{\prime}}\right\rangle=\sum_{j, j^{\prime}} \int_{\Omega^{\circ}} \widetilde{m}_{j}(\zeta) \overline{\widetilde{m}_{j^{\prime}}}(\zeta)\left(\mathbf{U}^{-1}\right)_{j j^{\prime}}(\zeta) d \zeta=\sum_{j} \int_{\Omega^{\circ}} B_{j}(\zeta) \overline{\widetilde{m}_{j}}(\zeta) d \zeta .
$$

Hence, we have

$$
\left\|\eta_{T}-\sum_{j} \widetilde{m}_{j} \mathscr{V}_{j}\right\|^{2}=\left\|\eta_{T}\right\|^{2}-\int_{\Omega^{\circ}} \sum_{j, j^{\prime}}\left(\mathbf{U}^{-1}\right)_{j j^{\prime}}(\zeta) B_{j}(\zeta) \overline{B_{j^{\prime}}}(\zeta) d \zeta
$$

and since $\left\|\eta_{T}\right\|^{2}=\int_{\Omega^{\circ}} \Pi^{\circ}\left(\left|\eta_{T}\right|^{2}\right)$ we obtain the error expression as stated.

Notice that Proposition 1 covers the Gabor multiplier case obtained in [7]. Notice also that (6) immediately yields

$$
E \leq\left\|\eta_{T}\right\|^{2}\left\|1-\frac{\sum_{j, j^{\prime}}\left(\mathbf{U}^{-1}\right)_{j j^{\prime}} B_{j} \overline{B_{j^{\prime}}}}{\Gamma_{T}}\right\|_{\mathbf{L}^{\infty}\left(\Omega^{\circ}\right)}
$$

The finite-dimensional situation is similar, the integral over $\Omega^{\circ}$ is replaced by a finite sum over the finite fundamental domain $\left\{0, \ldots t_{0}-1\right\} \times\left\{0, \ldots \xi_{0}-1\right\}$. We can now consider the case of an operator $T$ for which $\operatorname{supp}\left(\eta_{T}\right) \subseteq \Omega^{\circ}$ and obtain the following result. 
Corollary 1. Let an operator $T$ with $\operatorname{supp}\left(\eta_{T}\right) \subseteq \Omega^{\circ}$ be given. Then, the error of the best approximation by a generalized Gabor multiplier $G_{\mathbf{m}}$ as defined in (5) is bounded by

$$
\left\|T-G_{\mathbf{m}}\right\|_{H S}^{2} \leq\left\|\eta_{T}\right\|^{2} \underset{\zeta \in \Omega_{T}^{\circ}}{\operatorname{ess} \sup ^{\circ}}\left[\left|1-\left\langle\mathbf{U}(\zeta)^{-1} \cdot \mathbf{V}(\zeta), \mathbf{V}(\zeta)\right\rangle_{\mathbb{C}^{J}}\right|\right]
$$

where the vector-valued function $\mathbf{V}(\zeta)$ is given by $\mathbf{V}_{j}(\zeta)=\mathscr{V}_{g} h^{(j)}(\zeta)$ and $\Omega_{T}^{\circ}=$ $\Omega^{\circ} \cap \operatorname{Supp}\left(\eta_{T}\right)$.

Proof. Since $\operatorname{supp}\left(\eta_{T}\right) \subseteq \Omega^{\circ}$, we have $\mathbf{B}(\zeta)=\eta_{T}(\zeta) \cdot \mathbf{V}(\zeta)$ and the result follows from Proposition 1.

The last result may be interpreted as follows. For all $\zeta \in \Omega_{T}^{\circ}$, the matrix $\mathbf{U}(\zeta)^{-1}$ is the Gramian matrix of the vectors $\mathbf{V}_{j}^{\zeta}$ defined by their entries $\mathbf{V}_{j}^{\zeta}\left(\lambda^{\circ}\right)=\mathscr{V}_{g} h^{(j)}\left(\zeta+\lambda^{\circ}\right)$ for $\lambda^{\circ} \in \Lambda^{\circ}$. The inverse of the Gramian provides the biorthogonal system to this family of vectors, $\widetilde{\mathbf{V}_{j}^{\zeta}}=\left[\mathbf{U}(\zeta)^{-1} \cdot \mathbf{V}^{\zeta}\right]_{j}$, for every $\zeta \in \Omega_{T}^{\circ}$. Since, now, the support of $\eta_{T}$ is restricted to $\Omega_{T}^{\circ}$ in the present case, the vector $\eta_{T}^{\zeta}$, given by $\eta_{T}^{\zeta}\left(\lambda^{\circ}\right)=\eta_{T}\left(\zeta+\lambda^{\circ}\right), \lambda^{\circ} \in \Lambda^{\circ}$, to be approximated by the family of vectors $\mathbf{V}_{j}^{\zeta}, j=1, \ldots, J$, has only one non-zero entry, at $\lambda^{\circ}=0$. By realization of the matrix multiplication corresponding to the projection of $\eta_{T}^{\zeta}$ onto the span of the $\mathbf{V}_{j}^{\zeta}$, we find that requiring equality of $\eta_{T}^{\zeta}$ to its projection leads to the necessary condition that $\sum_{j} \widetilde{\mathbf{V}_{j}^{\zeta}}(0) \cdot \mathbf{V}_{j}^{\zeta}(0)=1$, which is equivalent to saying that $\left\langle\mathbf{U}(\zeta)^{-1} \cdot \mathbf{V}(\zeta), \mathbf{V}(\zeta)\right\rangle_{\mathbb{C}^{J}}$ is equal to 1 , for all $\zeta \in \Omega_{T}^{\circ}$.

Consequently, we have to find a family of windows that exhaust $\Omega_{T}^{\circ}$ in the sense of maximal concentration inside $\Omega_{T}^{\circ}$. Note that the concentration may (and will) be achieved by using functions with support outside $\Omega_{T}^{\circ}$ in order to cancel the aliases caused by the unavoidable energy of each $\mathbf{V}_{j}$ outside this area. We will come back to this situation in an example in Section 5.2.

The next corollary generalizes the result from the previous corollary. We assume that the spreading function is supported in a finite union of translates of the fundamental domain $\Omega^{\circ}$. In other words, we assume that $\eta(\zeta)=\sum_{l=1}^{L} \eta_{l}(\zeta)$ with $\operatorname{supp}\left(\eta_{l}\right) \subseteq\left(\Omega^{\circ}+\mu_{l}\right)$ for $l=1, \ldots, L$.

Corollary 2. Let an operator $T$ with $\operatorname{supp}\left(\eta_{T}\right) \subseteq \bigcup_{l=1}^{L}\left(\Omega^{\circ}+\mu_{l}\right)$ be given. Then, the error $E=\left\|T-G_{\mathbf{m}}\right\|_{H S}^{2}$ of the best approximation by a generalized Gabor multiplier $G_{\mathbf{m}}$ as defined in (5) is given by

$$
E=\int_{\Omega^{\circ}} \Gamma_{T}(\zeta)\left[1-\frac{\sum_{l=1}^{L}\left|\eta_{l}\left(\zeta+\mu_{l}\right)\right|^{2}\left\langle\mathbf{U}(\zeta)^{-1} \cdot \mathbf{V}\left(\zeta+\mu_{l}\right), \mathbf{V}\left(\zeta+\mu_{l}\right)\right\rangle}{\sum_{l=1}^{L}\left|\eta_{l}\left(\zeta+\mu_{l}\right)\right|^{2}}\right] d \zeta,
$$

where the vector-valued function $\mathbf{V}$ is given by $\mathbf{V}_{j}=\mathscr{V}_{g} h^{(j)}$ and $\eta_{l}=\eta_{T} \cdot \chi_{\Omega^{\circ}+\mu_{l}}$ is the restriction of $\eta_{T}$ to $\Omega^{\circ}+\mu_{l}$. 
Proof. According to the assumption on $\eta_{T}$, we may write $\eta_{T}=\sum_{l=1}^{L} \eta_{l}$, with $\operatorname{supp}\left(\eta_{l}\right) \subseteq\left(\Omega^{\circ}+\mu_{l}\right)$. Therefore, we obtain

$$
\begin{aligned}
B_{i}(\zeta) \overline{B_{j}}(\zeta) & =\sum_{k}\left(\eta_{T} \overline{\mathscr{V}_{g} h^{(i)}}\left(\zeta+\mu_{k}\right)\right) \cdot \sum_{l}\left(\overline{\eta_{T}} \mathscr{V}_{g} h^{(j)}\left(\zeta+\mu_{l}\right)\right) \\
& \left.=\sum_{l=1}^{L}\left|\eta_{l}\left(\zeta+\mu_{l}\right)\right|^{2} \overline{\left(\mathscr{V}_{g} h^{(i)}\right.} \cdot \mathscr{V}_{g} h^{(j)}\right)\left(\zeta+\mu_{l}\right),
\end{aligned}
$$

since the $\eta_{l}$ have, by definition, disjoint support. Hence, the result follows directly from (6).

Note that the last corollary shows that for sufficiently disjoint compactly supported regions $\Omega^{\circ}+\mu_{l}, l=1, \ldots, J$, of a given spreading function, the situation is comparable to the situation observed in Corollary 1. One synthesis window or, in an improved situation, a finite set of synthesis windows, should be concentrated as well as possible in each of the $\Omega^{\circ}+\mu_{l}$ and close to zero outside. On the other hand, in the case of adjacent $\Omega^{\circ}+\mu_{l}$, a similar strategy can be envisaged, with the virtue of mutual cancellation of aliases arising in each of the $\Omega^{\circ}+\mu_{l}$.

The next corollary deals with the approximation error occurring in the case of approximating an STFT multiplier by a Gabor multiplier. This is a situation of particular relevance, since in practice, the multiplier symbol $m$, originally defined on $\mathbb{R}^{2}$, is simply sub-sampled. Therefore, we also compare the result of this procedure to the corresponding best approximation. Let us recall that a short time Fourier multiplier is defined in a similar way as a Gabor multiplier, using pointwise multiplication in the time-frequency domain. In order to avoid confusion, we shall denote by $S_{a}$ a short time Fourier multiplier, defined by

$$
S_{a}: f \in \mathcal{H} \longmapsto S_{a} f=\mathscr{V}_{h}^{*}\left(a \cdot \mathscr{V}_{g} f\right),
$$

where $\mathscr{V}_{g}$ and $\mathscr{V}_{h}$ denote short time Fourier transforms with respect to windows $g$ and $h$ respectively, and the symbol, denoted by $a$, is now defined as a function on the whole time-frequency space (instead of a sub-lattice $\Lambda$ of it).

We recall [7], that the spreading function of an STFT-multiplier is given by $\eta_{S_{a}}=\widetilde{a} \cdot \mathscr{V}_{g} h, \widetilde{a}$ being the continuous symplectic Fourier transform of $a$.

Corollary 3. Let $T=S_{a}$ be a STFT multiplier with spreading function $\eta_{T}=$ $\widetilde{a} \cdot \mathscr{V}_{g} h$, and denote by $T^{\prime}=G_{m}$ its best Gabor multiplier approximation with the same windows, and lattice $\Lambda$, as defined in (5).

1. The approximation error is given by

$$
\left\|T-T^{\prime}\right\|_{\mathcal{H} S}^{2}=\int_{\Omega^{\circ}}\left[\Pi^{\circ}\left(\left|\left(\widetilde{a} \cdot \mathscr{V}_{g} h\right)\right|^{2}\right)(\zeta)-\frac{\left|\Pi^{\circ}\left(\widetilde{a} \cdot\left|\mathscr{V}_{g} h\right|^{2}\right)(\zeta)\right|^{2}}{\Pi^{\circ}\left(\left|\mathscr{V}_{g} h(\zeta)\right|^{2}\right)}\right] d \zeta
$$


2. Furthermore, the difference between the best approximation $T^{\prime}$ and the $G a$ bor multiplier $T^{\prime \prime}=G_{a \mid \Lambda, \Lambda}$ obtained from the $\Lambda$-subsampled version a $\mid \Lambda$ of the symbol a is given by

$$
\left\|T^{\prime \prime}-T^{\prime}\right\|_{\mathcal{H} \mathcal{S}}^{2}=\int_{\Omega^{\circ}}\left|\Pi^{\circ}(\widetilde{a})(\zeta)\right|^{2} \Pi^{\circ}\left(\left|\mathscr{V}_{g} h\right|^{2}\right)(\zeta)\left|1-\frac{\Pi^{\circ}\left(\widetilde{a} \cdot\left|\mathscr{V}_{g} h\right|^{2}\right)(\zeta)}{\Pi^{\circ}(\widetilde{a})(\zeta) \Pi^{\circ}\left(\left|\mathscr{V}_{g} h\right|^{2}\right)(\zeta)}\right|^{2} d \zeta
$$

Proof. To prove (9), we first note that, since the spreading function of $T^{\prime}=G_{m}$ is given by $\widetilde{m} \cdot \mathscr{V}_{g} h$, with $\widetilde{m}$ given in (5), we have

$$
\begin{aligned}
\left\|T-T^{\prime}\right\|_{\mathcal{H} S}^{2}= & \int_{\mathbb{R}^{2}}\left|\left[\widetilde{a}(\zeta)-\frac{\Pi^{\circ}\left(\widetilde{a} \cdot\left|\mathscr{V}_{g} h\right|^{2}\right)(\zeta)}{\Pi^{\circ}\left(\left|\mathscr{V}_{g} h(\zeta)\right|^{2}\right)}\right] \cdot \mathscr{V}_{g} h(\zeta)\right|^{2} d \zeta \\
= & \int_{\mathbb{R}^{2}}\left[\left|\left(\widetilde{a} \cdot \mathscr{V}_{g} h\right)(\zeta)\right|^{2}+\frac{\left|\Pi^{\circ}\left(\widetilde{a} \cdot\left|\mathscr{V}_{g} h\right|^{2}\right)(\zeta)\right|^{2}}{\left|\Pi^{\circ}\left(\left|\mathscr{V}_{g} h(\zeta)\right|^{2}\right)\right|^{2}} \cdot\left|\mathscr{V}_{g} h(\zeta)\right|^{2}\right. \\
& \left.\quad-2 \operatorname{Re}\left(\left(\overline{\widetilde{a}} \cdot\left|\mathscr{V}_{g} h\right|^{2}\right)(\zeta) \cdot \frac{\Pi^{\circ}\left(\left(\widetilde{a} \cdot\left|\mathscr{V}_{g} h\right|^{2}\right)(\zeta)\right)}{\Pi^{\circ}\left(\left|\mathscr{V}_{g} h(\zeta)\right|^{2}\right)}\right)\right] d \zeta .
\end{aligned}
$$

We then immediately obtain (9) since, by the usual periodization argument,

$$
\int_{\mathbb{R}^{2}}\left(\overline{\widetilde{a}} \cdot\left|\mathscr{V}_{g} h\right|^{2}\right)(\zeta) \cdot \frac{\Pi^{\circ}\left(\widetilde{a} \cdot\left|\mathscr{V}_{g} h\right|^{2}(\zeta)\right)}{\Pi^{\circ}\left(\left|\mathscr{V}_{g} h(\zeta)\right|^{2}\right)} d \zeta=\int_{\Omega^{\circ}} \frac{\left|\Pi^{\circ}\left(\widetilde{a} \cdot\left|\mathscr{V}_{g} h\right|^{2}(\zeta)\right)\right|^{2}}{\Pi^{\circ}\left(\left|\mathscr{V}_{g} h(\zeta)\right|^{2}\right)} d \zeta
$$

To see (10), note that the symplectic Fourier transform of $\left.a\right|_{\Lambda}$ is just the periodization of $\widetilde{a}$ on $\Lambda^{\circ}$. Hence, we have

$$
\begin{aligned}
\left\|T^{\prime \prime}-T^{\prime}\right\|_{\mathcal{H} \mathcal{S}}^{2} & =\int_{\mathbb{R}^{2}}\left|\Pi^{\circ}(\widetilde{a})(\zeta)-\frac{\Pi^{\circ}\left(\widetilde{a} \cdot\left|\mathscr{V}_{g} h\right|^{2}\right)(\zeta)}{\Pi^{\circ}\left(\left|\mathscr{V}_{g} h\right|^{2}\right)(\zeta)}\right|^{2} \cdot\left|\mathscr{V}_{g} h(\zeta)\right|^{2} d \zeta \\
& =\int_{\mathbb{R}^{2}}\left|\Pi^{\circ}(\widetilde{a})(\zeta) \Pi^{\circ}\left(\left|\mathscr{V}_{g} h\right|^{2}\right)(\zeta)-\Pi^{\circ}\left(\widetilde{a} \cdot\left|\mathscr{V}_{g} h\right|^{2}\right)(\zeta)\right|^{2} \cdot \frac{\left|\mathscr{V}_{g} h(\zeta)\right|^{2}}{\left(\Pi^{\circ}\left(\left|\mathscr{V}_{g} h\right|^{2}\right)(\zeta)\right)^{2}} d \zeta \\
& =\int_{\Omega^{\circ}} \frac{\left|\Pi^{\circ}(\widetilde{a})(\zeta) \cdot \Pi^{\circ}\left(\left|\mathscr{V}_{g} h\right|^{2}\right)(\zeta)-\Pi^{\circ}\left(\widetilde{a} \cdot\left|\mathscr{V}_{g} h\right|^{2}\right)(\zeta)\right|^{2}}{\Pi^{\circ}\left(\left|\mathscr{V}_{g} h(\zeta)\right|^{2}\right)} d \zeta
\end{aligned}
$$

which proves (10).

It becomes obvious that the error between the result obtained from subsampling, is insignificant as long as the symbol's (symplectic) Fourier transform $\widetilde{a}$ is concentrated near the origin. If higher frequencies are present in $a$, simple subsampling of the multiplier may lead to undesirable aliasing effects, as shown in the following simple example.

Example 1. Consider the case $\mathcal{H}=\mathbf{L}^{2}(\mathbb{R})$ with a separable lattice $\Lambda=$ $b_{0} \mathbb{Z} \times \nu_{0} \mathbb{Z}$, and let $S_{a}$ be an STFT multiplier with a symbol consisting of a 
low-frequency component and a higher-frequency perturbation component. For example, let $a$ be given by its symplectic Fourier transform $\widetilde{a}$ with $\widetilde{a}(\zeta)=1$ for $\zeta \in[-\varepsilon, \varepsilon] \times[-\varepsilon, \varepsilon]$ and $\widetilde{a}(\zeta)=-1$ for $\zeta \in\left[\frac{k_{1}}{\nu_{0}}-\varepsilon, \frac{k_{1}}{\nu_{0}}+\varepsilon\right] \times\left[\frac{k_{1}}{b_{0}}-\varepsilon, \frac{k_{1}}{b_{0}}+\varepsilon\right]$ for some integer $k_{1}, \varepsilon$ close to zero, and $\widetilde{a}(\zeta)=0$ otherwise. Then, if $\Lambda=b_{0} \mathbb{Z} \times \nu_{0} \mathbb{Z}$, obviously $\Pi^{\circ}(\widetilde{a})(\zeta) \equiv 0$, such that subsampling the original multiplier $a$ results in the zero operator. On the other hand, assuming an exponential decay for $\left|\mathscr{V}_{g} h\right|$, e.g. $\left|\mathscr{V}_{g} h(\zeta)\right|^{2} \leq e^{-|\zeta|^{4}}$, we have, with $\zeta_{1}=\left(\frac{k_{1}}{\nu_{0}}, \frac{k_{1}}{b_{0}}\right): \Pi^{\circ}\left(\widetilde{a} \cdot\left|\mathscr{V}_{g} h\right|^{2}\right)(\zeta) \approx 1-e^{-\left|\zeta_{1}\right|^{4}}$, for $\zeta \in\left[\frac{k}{\nu_{0}}-\varepsilon, \frac{k}{\nu_{0}}+\varepsilon\right] \times\left[\frac{k}{b_{0}}-\varepsilon, \frac{k}{b_{0}}+\varepsilon\right], k \in \mathbb{Z}$, and $\Pi^{\circ}\left(\widetilde{a} \cdot\left|\mathscr{V}_{g} h\right|^{2}\right)(\zeta)=0$ else. So, in this case, the aliases that would be generated by subsampling are efficiently suppressed in the approximation.

The above example shows that knowing some properties of a system can help to choose appropriate parameters in the approximation of the corresponding operators. For example, if an STFT multiplier is generated by a strictly lowpass symbol, sub-sampling the symbol is a good choice. As soon as the symbol has higher-frequency components, this may result in quite different operators and best approximation of the original system should be preferred. Similar observations can be made for alternating between different lattices. The next section discusses the choice of parameters in more detail.

\section{Choosing the parameters}

For simplicity, we specialize the following discussion to the infinite-dimensional case $\mathcal{H}=\mathbf{L}^{2}(\mathbb{R})$, and rectangular lattice $\Lambda=b_{0} \mathbb{Z} \times \nu_{0} \mathbb{Z}$. The finite-dimensional situation is handled similarly.

\subsection{Gabor Multipliers}

If an operator $T$ with known spreading function is to be approximated by a Gabor multiplier $G_{m}$, the lattice may be adapted to the eccentricity of the spreading function according to the error expression obtained in Proposition 1, which may be considerably simplified for the case of only one synthesis window, see [7]. In order to choose the eccentricity of the lattice accordingly and adapt the window to the chosen lattice as to avoid aliasing, assume, that we may find $b_{0}, \nu_{0}$, with $b_{0} \cdot \nu_{0}<1$, such that $\operatorname{supp}\left(\eta_{T}\right) \subseteq\left(\Omega^{\circ}+z\right)$, where $\Omega^{\circ}=\left[0, \frac{1}{\nu_{0}}\right] \times\left[0, \frac{1}{b_{0}}\right]$. In this case, the error resulting from best approximation by a Gabor multiplier with respect to the lattice $\Lambda=b_{0} \mathbb{Z} \times \nu_{0} \mathbb{Z}$ is bounded by $K_{e} \cdot\left\|\eta_{T}\right\|_{2}^{2}$, with

$$
K_{e}=1-\inf _{t, \xi \in \Omega_{T}^{\circ}} \frac{\left|\mathscr{V}_{g} h(t, \xi)\right|^{2}}{\sum_{k, l}\left|\mathscr{V}_{g} h\left(t+k / \nu_{0}, \xi+l / b_{0}\right)\right|^{2}},
$$

with $\Omega_{T}^{\circ}=\Omega^{\circ} \cap \operatorname{Supp}\left(\eta_{T}\right)$. Optimal results are therefore expected if $g, h$ can be chosen in such a way that $K_{e}$ be minimum, for a given lattice, i.e. when the 
cross-ambiguity function $\mathscr{V}_{g} h$ is "concentrated" inside the fundamental domain $\Omega^{\circ}$. It is worth noticing that such a concentration property, which may be seen as a lattice-constrained time-frequency uncertainty, differs from what is usually required in, e.g. radar applications, since the geometry of the adjoint lattice has to be accounted for. Heuristically as well as from numerical experiments we know, that the tight window, [12], corresponding to a given lattice and a reasonably localized window is usually a good choice to fulfill this requirement, since this window automatically adapts to the eccentricity of the lattice. On the other hand, a window that is better concentrated inside $\Omega^{\circ}$, may be obtained by computing the eigenfunction corresponding to the biggest eigenvalue of the time-frequency localization operator corresponding to $\Omega^{\circ}$. This operator is given as an STFT multiplier

$$
S_{\chi_{\Omega^{\circ}}}=\mathscr{V}_{g}^{*} \chi_{\Omega^{\circ}} \mathscr{V}_{g},
$$

$\chi_{\Omega^{\circ}}$ denoting the indicator function of the fundamental domain $\Omega^{\circ}$. Timefrequency localization operators have been studied extensively, e.g., [16, 3, 4, $13,5]$. Of course, this approach is less straight-forward and involves considerable computational effort. However, it may be generalized, as we will explain in the next section.

\subsection{Multiple and generalized Gabor Multipliers}

The main additional task in the generalized situation is the choice of the (additional) synthesis windows, or, in case only time-frequency shifts of one synthesis window are considered (generalized Gabor Multipliers), the choice of sampling points $\mu_{j}$ for the (additional) synthesis windows. A good choice will again be guided by the behavior of the spreading function.

Even for operators with a spreading function with compact support inside $\Omega^{\circ}$, considerable aliasing effects caused by the periodization of $\widetilde{a}$ have to be taken into account. It turns out that choosing a few eigenfunctions of $S_{\chi_{\Omega^{\circ}}}$ can significantly reduce the amount of aliasing in the approximation of underspread operators by generalized Gabor multipliers. This idea was already noted in [17] in a slightly different context. It becomes now obvious from Corollary 1, why these particular functions, by their property of being maximally concentrated inside $\Omega^{\circ}$, represent a good choice for the synthesis windows of multiple Gabor multipliers.

When dealing with overspread operators, the additional energy of the operator's spreading function outside $\Omega^{\circ}$ poses the second source of error on top of aliasing. The relevant areas in the spreading domain should be covered as well as possible with the smallest possible "leakage" beyond $\Omega^{\circ}$ of the different synthesis windows' cross-ambiguity functions. Motivated by the results from the Gabor multiplier situation, we choose a tight window with respect to the analysis lattice and look for the most appropriate sampling points for the synthesis 
windows. Examples will be given in Section 5.2.

Alternatively, one or several eigenfunctions of $S_{\chi_{\Omega^{\circ}}}$ may be time-frequency shifted to cover the areas in phase space where significant energy of the operator's spreading function occurs.

\section{$5 \quad$ Examples}

We now turn to numerical experiments, in the finite case $\mathcal{H}=\mathbb{C}^{L}$. In the following examples, the relative approximation error for the best approximation $T^{\prime}=G_{m}$ of $T$ is measured by the logarithmic quantity

$$
E=\log _{10}\left(\left\|T-G_{m}\right\| /\|T\|\right)
$$

where $\|\cdot\|$ represents a generic matrix norm. We display below the results obtained using the Frobenius norm, the plots obtained with the operator norm are almost identical.

\subsection{Classical Gabor Multipliers}

Experiment 1: We generate operators with compact support in the spreading domain, in a square of side size between 3 and 61 , symmetric about 0 . The values are complex, uniformly distributed random numbers, the signal length is $L=180$. We then investigate the approximation quality for various pairs of lattice constants, with $b_{0}$ varying between 2 and 18 and $\nu_{0}$ between 2 and 10 . The results, averaged over 40 realizations of the random spreading functions, are presented in Figure 2. Evidently, the error decreases for decreasing $b_{0}$, in each plot. Note the two distinct regimes: the error grows exponentially (linearly in the logarithmic representation of the figure) up to a certain value of the support size, depending on the lattice density, and slower thereafter. The explanation for this effect is the observation, that the error (see the bound in (11)) is comprised of an aliasing error and the inherent inaccuracy of Gabor multiplier approximation, even for very high sampling density, of overspread operators.

Experiment 2: In order to emphasize the importance of lattice adaptation to eccentricity of the spreading function's support, we show the results for different lattice constants resulting in the same redundancy (5) in Figure 3. The solid lines show the results for $b_{0}=\nu_{0}=6$, leading to far better results than the lattice constants not adapted to the (symmetric) support of the spreading function.

\subsection{Generalized Gabor Multipliers}

Experiment 3: We now investigate the influence of additional synthesis windows on the approximation quality. We first consider the same operators as in the previous section, but allow for additional synthesis windows. We compare 

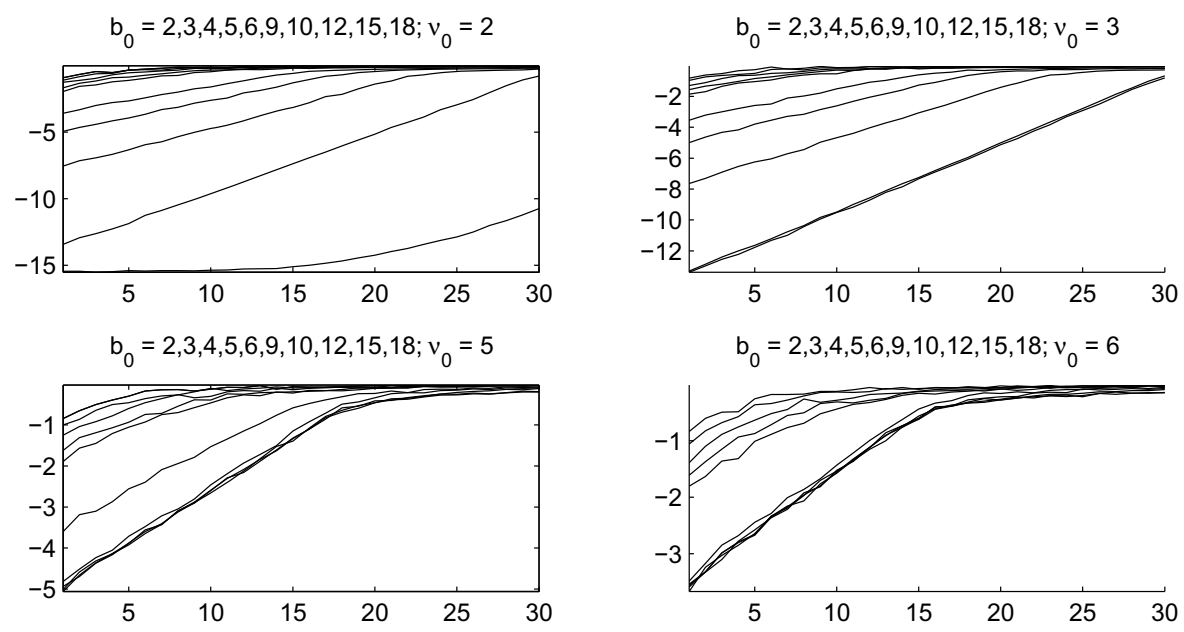

$\mathrm{b}_{0}=2,3,4,5,6,9,10,12,15,18 ; v_{0}=9$
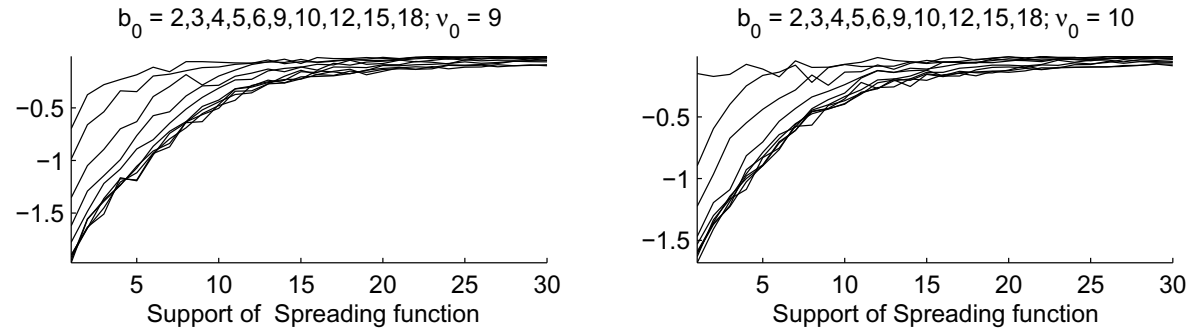

Figure 2: Logarithmic approximation error for different bandwidth of spreading function and different values of $b_{0}, \nu_{0}$

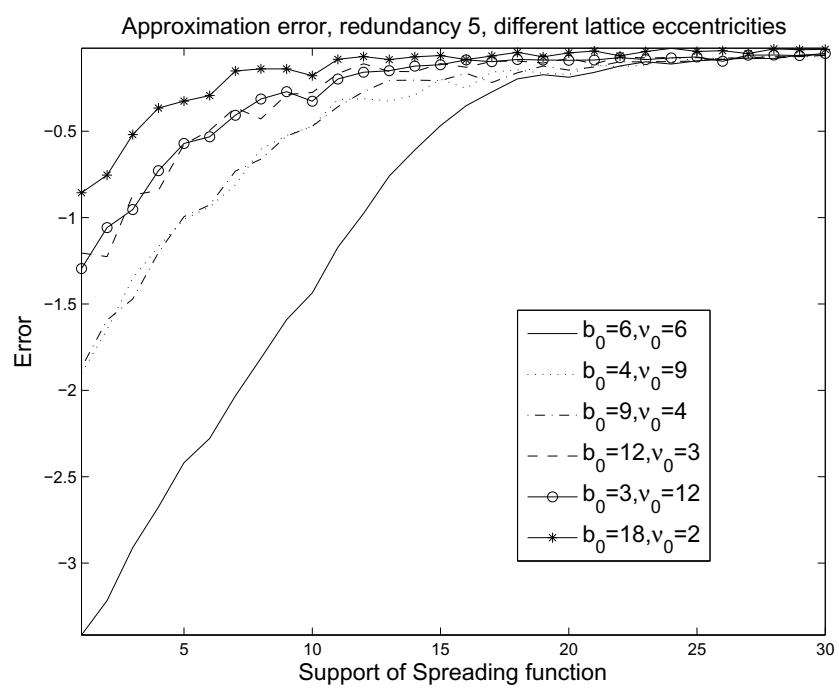

Figure 3: Logarithmic approximation error for different lattice-eccentricity 

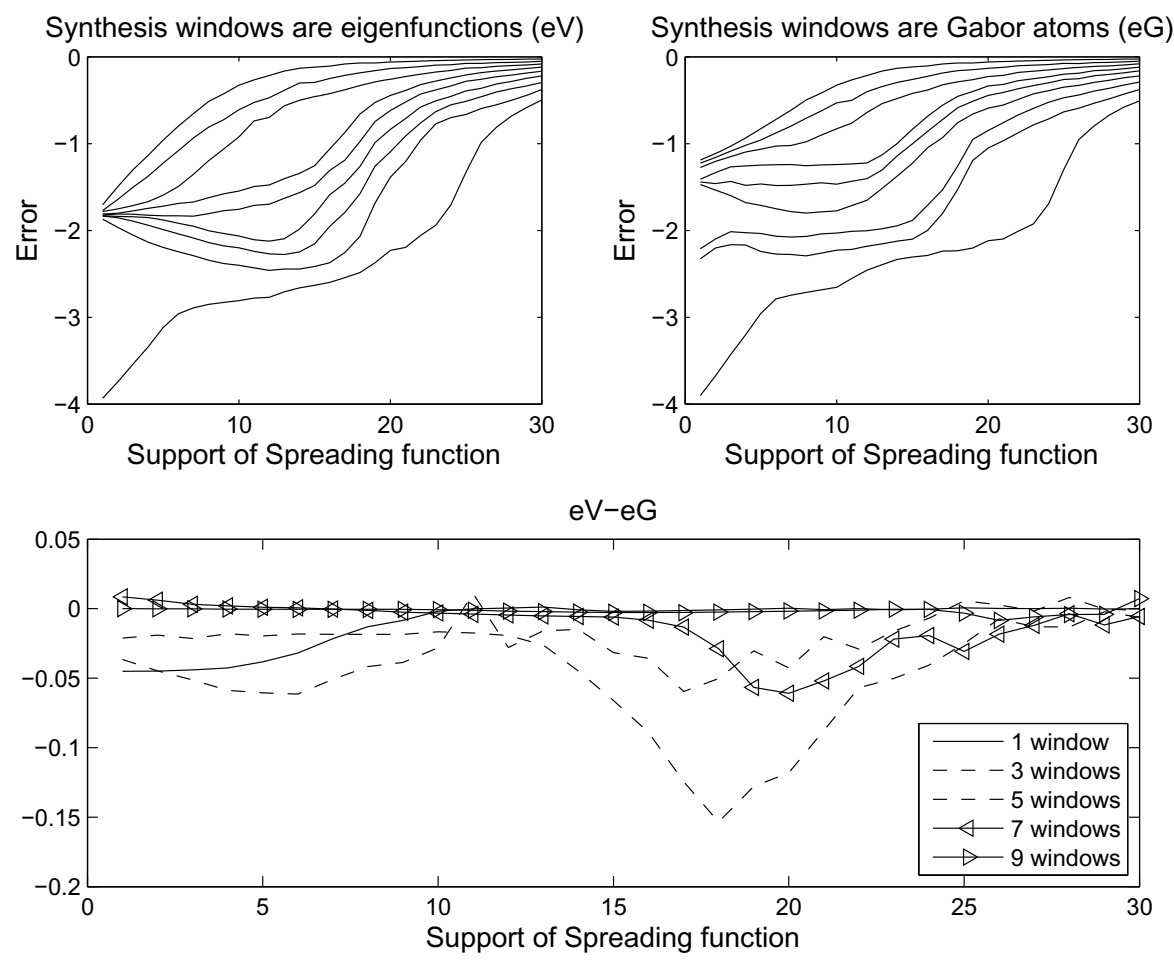

Figure 4: Logarithmic approximation error for growing support of spreading function, comparing eigenfunctions and Gabor atoms as synthesis windows

the performance of generalized Gabor multipliers and multiple Gabor multipliers. We use up to 9 synthesis windows generated as time-frequency shifted copies of the tight window corresponding to $\Lambda=9 \mathbb{Z} \times 9 \mathbb{Z}$. The sampling points are chosen from the intersection of $\Omega^{\circ}$ with $\Lambda$. On the other hand, we choose up to 9 eigenvectors of the time-frequency localization operator $S_{\chi_{\Omega^{\circ}}}$, using the eigenvectors corresponding to the biggest eigenvalues, in decreasing order. The results are displayed in Figure 4, where the two upper plots show the error for growing support of the spreading function. The errors for different numbers of synthesis windows are each plotted separately, the upper left display showing the errors resulting from the usage of eigenvectors $(e V)$, while $e G$ denotes the errors resulting from using Gabor atoms as synthesis windows. Obviously, additional synthesis windows improve the approximation quality. In order to better compare the performance of the two different sets of synthesis windows, the differences between the errors obtained in both cases are shown in the lower plot. The choice of eigenfunctions yields an over-all improvement of approximation quality, as expected from the analytic results. 

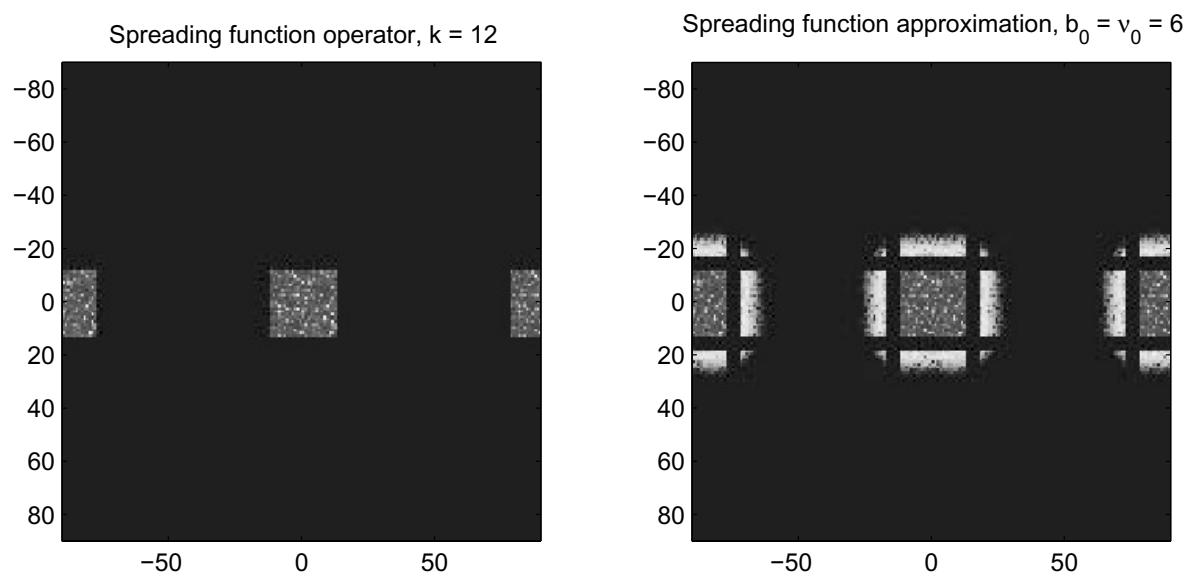

Figure 5: Spreading function of operator (experiment 4) and of best multiple Gabor multiplier approximation using two synthesis windows

Experiment 4: We investigate the following situation: an operator with two effectively disjoint components in the spreading domain is, again, approximated by a multiple Gabor multiplier with two synthesis windows. For better comparison, the two components are the component from the previous examples plus a shifted version (by 90 samples) thereof. Figure 5 shows the spreading functions of one of the operators and its best approximation with two synthesis windows, for the optimal additional window. Note the aliasing effect.

In this situation, using two appropriate synthesis windows, the obtained results are similar to those in the case of one spreading function component and one synthesis window, as discussed in the previous section. In Figure 6, we display the results for 3 symmetric pairs of lattice constants, the optimal window's result being represented by the solid line, while the dashed lines show the results of close but suboptimal synthesis windows. As the operator was generated by a translation by 90 samples, the tight window, shifted by 90 samples itself, is expected to be the optimal additional window. This is confirmed by the experiments. Analogously, the setting in Experiment 3 can be repeated for two (or more) disjoint components in the spreading domain, leading to largely comparable results.

Experiment 5: In a last experiment, we compare operators with a compact spreading function inside $\Omega^{\circ}$ (Operator 0), to operators with compact spreading function inside $\Omega^{\circ} \cup\left(\Omega^{\circ}+1 / \nu_{0}\right)$ (Operator 1 ) and $\Omega^{\circ} \cup\left(\Omega^{\circ}+2 / \nu_{0}\right)$ (Operator 2), respectively. In other words, in the first case, the two fundamental domains are adjacent and in the second case they are sufficiently separated. Again, the values of the operators' spreading function are random. We investigate the approximation quality for 1 to 6 eigenfunctions of $S_{\chi_{\Omega^{\circ}}}$ in case of Operator 0 , and 

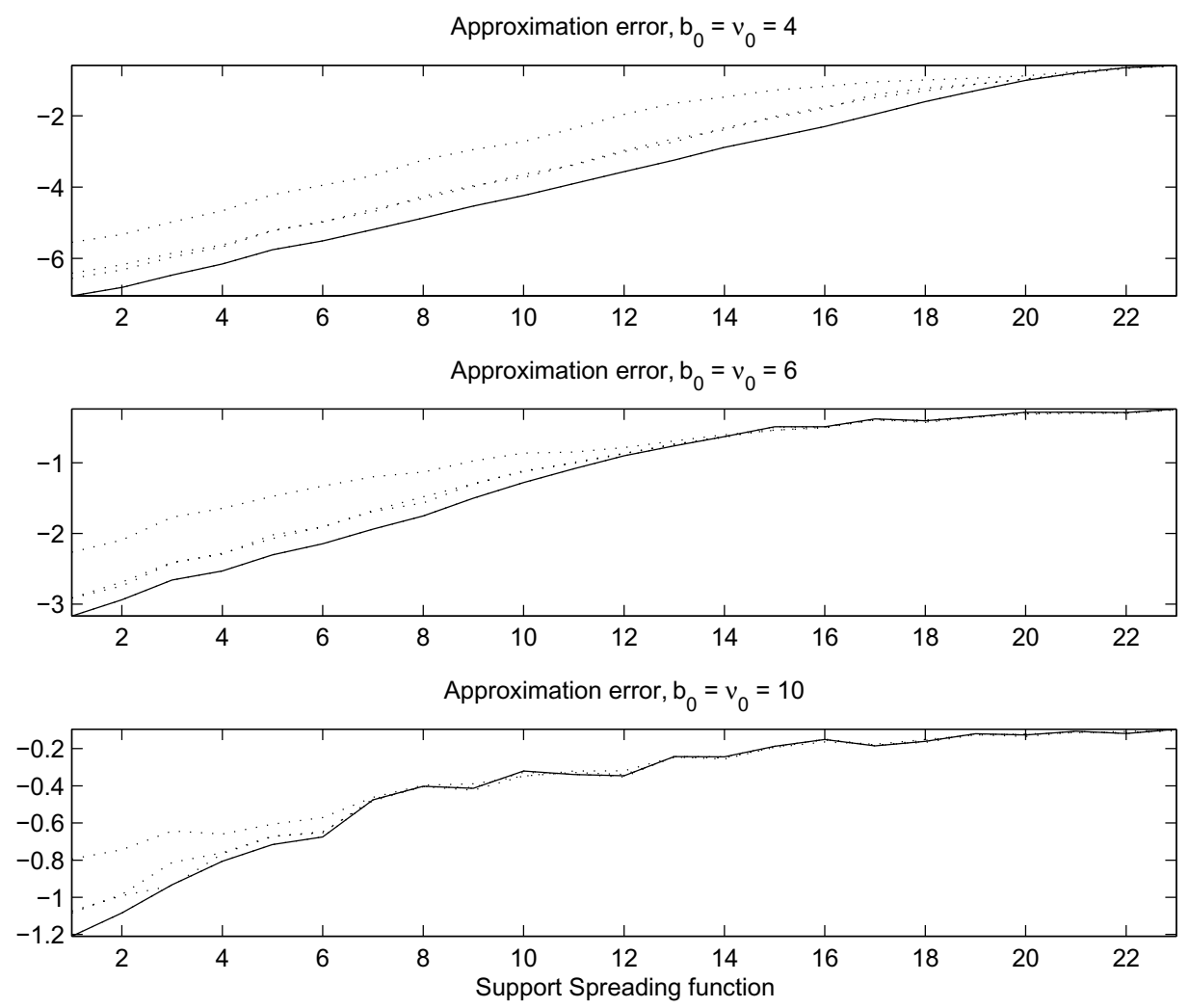

Figure 6: Approximation error for varying support of two components of spreading function and two synthesis windows

these eigenfunctions are, in each case, shifted to the appropriate fundamental domain. The lattice parameters were chosen to be $b_{0}=\nu_{0}=9$, so that, in this case, the support of the spreading functions lies in a (union of) fundamental domain $/ \mathrm{s}$ of $\Lambda^{\circ}$. This is the situation described in Corollary 2. Figure 7 shows the results of this experiment. The errors shown result from averaging over 40 different (random) spreading functions with the prescribed support.

As expected, the two disjoint spreading function components lead to largely comparable results. On the other hand, for two adjacent components, each of the components benefits from the eigenfunctions that are essentially concentrated in the neighboring fundamental domain. Apparently, this effect grows, when eigenfunctions less concentrated around 0 (i.e. corresponding to bigger eigenvalues) are added.

We remark, that the alternative idea to directly choose eigenfunctions corresponding to the bigger region $\Omega^{\circ} \cup\left(\Omega^{\circ}+1 / \nu_{0}\right)$ gives significantly poorer approximation results. 


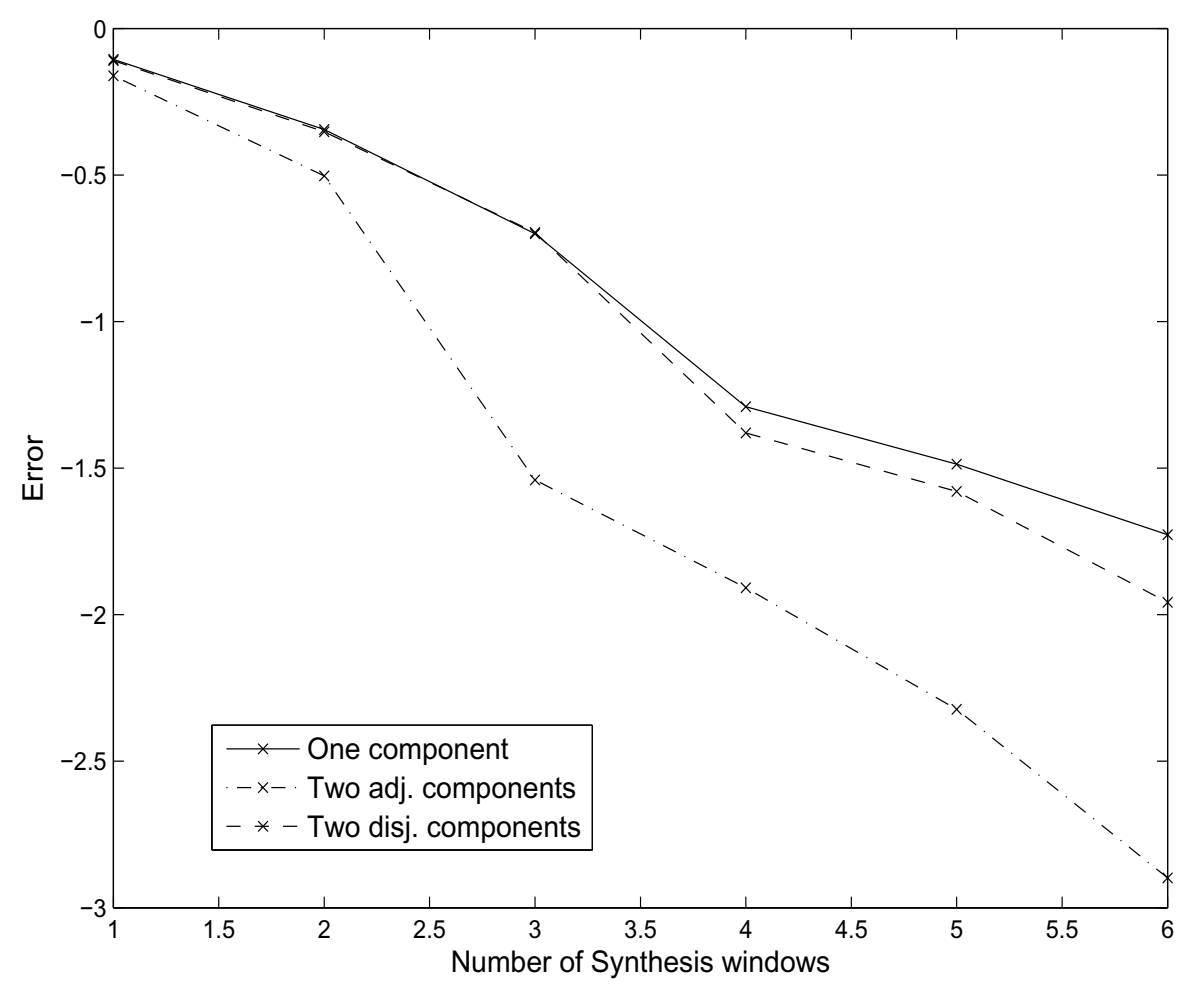

Figure 7: Comparing the approximation error for one or two spreading function components and 1 to 6 synthesis windows

\section{Discussion and conclusions}

The examples given in the previous section show that the choice of various parameters has considerable influence on the performance of approximation by (generalized) Gabor multipliers. While the situation is rather easily understood in the case of classical Gabor multipliers (at least qualitatively, though a better theoretical control would still be desirable), the generalized case involves a more subtle choice of additional parameters. Both the analytical results provided in Section 3 and the experiments from the previous section suggest that aliasing effects resulting from coarse sampling lattice can best be suppressed by using eigenfunctions of the localization operator $S_{\chi_{\Omega^{\circ}}}$ as additional synthesis windows. It should be noted that, while yielding better results in the approximation, using a small number of additional synthesis windows does not dramatically increase the computational cost: in (5), going from $|J|=1$ to larger index sets $J$ involves inverting (generally small) matrices instead of computing a point-wise ratio. Experiment 5 in the previous section shows that components of the spreading function of operators, that are not underspread, are very efficiently covered once 
the (approximate) location of these components is known. Hence, the development of an efficient method to estimate relevant components of the spreading function will be an important question in further research on the present topic.

\section{ACKNOWLEDGEMENT}

The first author was funded by the Austrian Science Fund (FWF) project LOCATIF(T384-N13). The second author acknowledges partial support from CNRS through the PEPS programme MTF\&Sons and from the European Union through the EU FET Open grant UNLocX (255931).

\section{References}

[1] P. Balazs, B. Laback, G. Eckel, and W. A. Deutsch, Time-Frequency Sparsity by Removing Perceptually Irrelevant Components Using a Simple Model of Simultaneous Masking, IEEE Transactions on Audio, Speech and Language Processing, 18(1), 34-49, 2010.

[2] J. Benedetto and G. Pfander, Frame expansions for Gabor multipliers, Applied and Computational Harmonic Analysis, 20(1), 26-40, 2006.

[3] E. Cordero and K. Gröchenig, On the product of localization operators. In M. Wong, editor, Modern Trends in Pseudo-differential Operators, volume 172 of Oper. Theory Adv. Appl., pages 279-295. Birkhäuser, Basel, 2007.

[4] E. Cordero, L. Rodino, and K. Gröchenig, Localization operators and timefrequency analysis. In N. Chong and et al., editors, Harmonic, Wavelet and p-adic Analysis, pages 83-110. World Sci. Publ., Hackensack, 2007.

[5] M. Dörfler and H. G. Feichtinger, Orthogonal projections derived from localization operators, Proc. Conf. EUSIPCO (Sept. 2004, TU Vienna), 1195-1198, 2004.

[6] M. Dörfler and B. Torrésani, Representation of operators by sampling in the time-frequency domain, Proceedings of SAMPTA09, 2009. http://hal.archives-ouvertes.fr/hal-00451424/fr/.

[7] M. Dörfler and B. Torrésani, Representation of operators in the timefrequency domain and generalized Gabor multipliers, J. Fourier Anal. Appl., 16(2), 261-293, 2010.

[8] H. G. Feichtinger, M. Hampejs, and G. Kracher, Approximation of matrices by Gabor multipliers, IEEE Signal Proc. Letters, 11(11), 883- 886, 2004. 
[9] H. G. Feichtinger and N. Kaiblinger, Varying the time-freqency lattice for Gabor frames, Transactions of the American Mathematical Society, 356(5), 2001-2023, 2003.

[10] H. G. Feichtinger and W. Kozek, Quantization of TF lattice-invariant operators on elementary LCA groups. In Gabor analysis and algorithms, pages 233-266. Birkhäuser Boston, Boston, MA, 1998.

[11] H. G. Feichtinger and K. Nowak, A first survey of Gabor multipliers. In H. G. Feichtinger and T. Strohmer, editors, Advances in Gabor Analysis, Boston, 2002. Birkhauser.

[12] H. G. Feichtinger and T. Strohmer, Gabor Analysis and Algorithms. Theory and Applications, Birkhäuser, 1998.

[13] C. Fernandez and A. Galbis, Compactness of time-frequency localization operators on $L^{2}(R)$, J. Funct. Anal., 233(2), 335-350, 2006.

[14] K. Gröchenig, Foundations of Time-Frequency Analysis, Appl. Numer. Harmon. Anal., Birkhäuser Boston, 2001.

[15] K. Gröchenig, Representation and approximation of pseudodifferential operators by sums of Gabor multipliers, Appl. Anal., 90(3-4), 385-401, 2011.

[16] K. Gröchenig and M. Dörfler, Time-Frequency partitions and characterizations of modulations spaces with localization operators, J. Funct. Anal., 260(7), 1903-1924, 2011.

[17] F. Hlawatsch, G. Matz, H. Kirchauer, and W. Kozek, Time-frequency formulation, design, and implementation of time-varying optimal filters for signal estimation, IEEE Trans. Signal Process., 48(5), 1417-1432, 2000.

[18] W. Kozek, Adaptation of Weyl-Heisenberg frames to underspread environments. In H. Feichtinger and T. Strohmer, editors, Gabor Analysis and Algorithms: Theory and Applications, pages 323-352. Birkhäuser Boston, 1997.

[19] Y. Li and D. Wang, On the optimality of ideal binary time-frequency masks, Speech Communication, 51(3), 230-239, 2009.

[20] A. Olivero, R. Kronland Martinet, and B. Torrésani, A new method for Gabor multiplier estimation: application to sound morphing, Proceedings of EUSIPCO 2010, August, 2010. http://hal.archives-ouvertes.fr/hal00493711/en/. 